\title{
New Approaches to Minimum-Energy Design of Integer- and Fractional-Order Perfect Control Algorithms
}

\author{
Wojciech P. Hunek ${ }^{1, *}$, and Eukasz Wach ${ }^{1}$ \\ ${ }^{1}$ Opole University of Technology, Institute of Control Engineering, Prószkowska 76, 45-758 Opole, Poland
}

\begin{abstract}
In this paper the new methods concerning the energy-based minimization of the perfect control inputs is presented. For that reason the multivariable integer- and fractional-order models are applied which can be used for describing a various real world processes. Up to now, the classical approaches have been used in forms of minimum-norm/least squares inverses. Notwithstanding, the above-mentioned tool do not guarantee the optimal control corresponding to optimal input energy. Therefore the new class of inversebased methods has been introduced, in particular the new $\sigma$ - and $H$-inverse of nonsquare parameter and polynomial matrices. Thus a proposed solution remarkably outperforms the typical ones in systems where the control runs can be understood in terms of different physical quantities, for example heat and mass transfer, electricity etc. A simulation study performed in Matlab/Simulink environment confirms the big potential of the new energy-based approaches.
\end{abstract}

\section{Introduction}

The issues involving the stability and robustness of $\mathrm{MV} /$ perfect control for LTI MIMO integer-order discrete-time systems in state-space framework become the field of intense scientific research [1-7]. Taking into account the integer-order perfect control the quest for advance takes the form of utilization different types of parameter of polynomial right inverses. Until now, the mostly used and widely presented in the literature is unique minimum-norm right $T$-inverse, regarded as minimum energy one $[1,4,5]$. However, application the unique $T$-inverse produce only one inverse model control (IMC) system, hence it cannot be used to wide class of cases where the control inputs remain unstable under perfect control law. To overcome such obstacle there is a need for finding nonunique right inverse letting us generate an infinite set of IMC systems, amongst which we can look for stable and robust ones. The first method can be application of newly introduced set of nonunique parameter and polynomial right inverses, in particular $\sigma$ - and $H$-inverse [3-5,8-9]. The crucial features of above-mentioned inverses are so-called degrees of freedom which effectively impact the robustness, especially in terms of minimum-energy perfect control design. Aforementioned exploration of new ways of enhancement the robustness of discrete-time integerorder perfect control systems, in authors opinion, can be also widen by implementation fractional-order model considered as the model closer to the real world objects, giving the prospect to develop more efficient i.e. lower energy consuming and more accurate control strategies. The method of employment fractional model is based on entering slight modification into the order $\alpha$ of well- known Grünwald-Letnikov discrete-time fractional difference operator $\Delta^{\alpha}$ of non-integer order $\alpha$ [10-11], which for classical perfect control systems is equal to $\alpha=1$. The gist of approach is such modification of the perfect control system order that do not distort the original system i.e. leaves parameter $\alpha$ value still close to $\alpha=1$ but influence its stability and robustness. In order to evaluate the outcome the energy-based performance indices necessary to assess the robustification of the perfect control, is introduced. The presented methodology indicates the direction to develop the new algorithms for minimizing the energy of fractional-order $\mathrm{MV} /$ perfect control inputs. Of course, the new method is mainly dedicated to nonsquare systems i.e. systems having different number of input and output variables.

\section{System representation}

Linear time-invariant (LTI) discrete-time fractionalorder system $\mathbf{S}\left(\mathbf{A}_{\mathbf{d}}, \mathbf{B}, \mathbf{C}\right)$ described by the GrünwaldLetnikov fractional difference operator is defined by the formula [10-11]

$\left\{\begin{array}{c}\Delta^{\alpha} \mathbf{x}(k+1)=\mathbf{A}_{\mathbf{d}} \mathbf{x}(k)+\mathbf{B u}(k) ; \mathbf{x}(0)=\mathbf{x}_{\mathbf{0}}, \\ \mathbf{y}(k)=\mathbf{C} \mathbf{x}(k)\end{array}\right.$

where $k$ denotes the discrete time, whereas GrünwaldLetnikov fractional difference operator $\Delta^{\alpha}$ of an order $\alpha$ $(0<\alpha<2, \alpha \in \mathbb{R})$ is defined by following formula:

$$
\Delta^{\alpha} \mathbf{x}(k)=\sum_{j=0}^{k}(-1)^{j}\left(\begin{array}{l}
\alpha \\
k
\end{array}\right) \mathbf{x}(k-j),
$$

whilst

\footnotetext{
* Corresponding author: w.hunek@po.opole.pl
} 


$$
\left(\begin{array}{l}
\alpha \\
j
\end{array}\right)= \begin{cases}1 & j=0 \\
\frac{\alpha(\alpha-1) \ldots(\alpha-j+1)}{j !} & j>0 .\end{cases}
$$

The system dimensions is defined as follows: $n_{u^{-}}$ inputs $\mathbf{u}(k), n_{\mathrm{y}}$-outputs $\mathbf{y}(k)$ and $n$-state vector $\mathbf{x}(k)$.

Remark 1. For classical state-space systems with $\alpha=1$ we have

$$
\left\{\begin{array}{l}
\mathbf{x}(k+1)=\mathbf{A x}(k)+\mathbf{B u}(k) ; \mathbf{x}(0)=\mathbf{x}_{\mathbf{0}} \\
\mathbf{y}(k)=\mathbf{C} \mathbf{x}(k)
\end{array}\right.
$$

where $\mathbf{A}=\mathbf{A}_{\mathbf{d}}+\mathbf{I}_{\boldsymbol{n}}$ and $\mathbf{I}_{\boldsymbol{n}}$ is the identity $n$-matrix.

\section{Integer-/fractional-order perfect control}

To consider the discrete-time perfect control for fractional-order system denoted by formulas (1-3) it is essential to determine and minimize the performance index described by the following formula

$$
\begin{gathered}
J(\mathbf{u}(k))=\sum_{k=0}^{\infty}\left[\mathbf{y}(k+1)-\mathbf{y}_{\text {ref }}(k+1)\right]^{\mathrm{T}} \\
{\left[\mathbf{y}(k+1)-\mathbf{y}_{\text {ref }}(k+1)\right],}
\end{gathered}
$$

in which $\mathbf{y}(k+1)$ represents one step deterministic output predictor and $\mathbf{y}_{\mathbf{r e f}}(k+1)$ indicates the one-step output reference/setpoint. This enables defining the fractional-order perfect control law or right-invertible systems [10]

$$
\begin{gathered}
\mathbf{u}(k)=(\mathbf{C B})^{\mathrm{R}}\left[\mathbf{y}_{\mathbf{r e f}}(k+1)-\mathbf{C A}_{\mathbf{d}} \mathbf{x}(k)\right. \\
\left.+\mathbf{C} \sum_{j=1}^{k+1}(-1)^{j}\left(\begin{array}{l}
\alpha \\
k
\end{array}\right) \mathbf{x}(k-j-1)\right] .
\end{gathered}
$$

For classical state-space systems with parameter $\alpha=1$ the Eqn. (6) reduces to the multivariable integer-order perfect control

$$
\mathbf{u}(k)=(\mathbf{C B})^{\mathrm{R}}\left[\mathbf{y}_{\mathbf{r e f}}(k+1)-\mathbf{C A}_{\mathbf{d}} \mathbf{x}(k)\right] .
$$

Remark 2. In further consideration are taken into account only state-space LTI MIMO discrete-time systems with product of CB being right invertible, where symbol ' $R$ ' denotes ant right inverse. This imposes that number of inputs of the object is higher than number of its outputs $\left(\mathrm{n}_{\mathrm{u}}>\mathrm{n}_{\mathrm{y}}\right)$. For such systems the perfect control exists [1]. For left-invertible systems $\left(\mathrm{n}_{\mathrm{u}}<\mathrm{n}_{\mathrm{y}}\right)$ the mentioned control strategy cannot be obtained. The issue concerning the implementation of nonsquare right inverses into perfect control laws is discussed in next section

\section{Inverses of nonsquare matrices}

At the beginning the definition of classical unique minimum-norm $T$-inverse of $\underline{\mathbf{B}}\left(q^{-1}\right)$ with dimension $n_{y} \times n_{u}$, dedicated to full normal rank systems in the backward shift operator domain, is presented [1].

Definition. Let the polynomial matrix $\underline{\mathbf{B}}\left(q^{-1}\right)=$ $\underline{\mathbf{b}}_{\mathbf{0}}+\underline{\mathbf{b}}_{\mathbf{1}} q^{-1}+\cdots+\underline{\mathbf{b}}_{\mathbf{m}} q^{-m}$ be of full rank $n_{y}$ (or $\left.n_{u}\right)$. The (unique) minimum-norm right (or least square left) $T$-inverse of $\underline{\mathbf{B}}\left(q^{-1}\right)$ is defined as
$\underline{\mathbf{B}}_{0}^{\mathrm{R}}\left(q^{-1}\right)=\underline{\mathbf{B}}^{\mathrm{T}}\left(q^{-1}\right)\left[\underline{\mathbf{B}}\left(q^{-1}\right) \underline{\mathbf{B}}^{\mathrm{T}}\left(q^{-1}\right)\right]^{-1} \quad\left(\right.$ or $\quad \underline{\mathbf{B}}_{0}^{\mathrm{L}}=$ $\left.\left[\underline{\mathbf{B}}\left(q^{-1}\right) \underline{\mathbf{B}}^{\mathrm{T}}\left(q^{-1}\right)\right]^{-1} \underline{\mathbf{B}}^{\mathrm{T}}\left(q^{-1}\right)\right)$.

For the purpose of further analysis the parameter system is given due consideration which forms a special instance of polynomial one under $\underline{\mathbf{B}}\left(q^{-1}\right)=\underline{\mathbf{b}}_{\mathbf{0}}$. However, the unique $T$-inverse generates exactly one solution, which in many cases do not meet the requirements of conducted study.

An enhancement of aforementioned unique right $T$ inverse is nonunique right $\sigma$-inverse involving so-called degrees of freedom. The recent formalization of $\sigma$ inverse is presented in Ref. [4] in form of Corollary 1.

Corollary. Let the polynomial matrix $\underline{\mathbf{B}}\left(q^{-1}\right)=\underline{\mathbf{b}}_{\mathbf{0}}+$ $\underline{\mathbf{b}}_{1} q^{-1}+\cdots+\underline{\mathbf{b}}_{\mathbf{m}} q^{-m}$ be of full normal rank $n_{y}$ (or $\left.n_{u}\right)$ and let $z^{s} \boldsymbol{\beta}\left(z^{-1}\right)=\boldsymbol{\beta}(z) \in \mathbb{R}^{n_{y} \times n_{u}}[z]$ be arbitrary, including an arbitrary order $s$. Assume additionally that the product $\underline{\mathbf{B}}\left(q^{-1}\right) \underline{\boldsymbol{\beta}}^{\mathrm{T}}\left(q^{-1}\right)$ is of full normal rank $n_{y}$ (or $n_{u}$ ). Then an old form of $\sigma$-inverse (see [1], p. 57, Eqn. (5.3)) can be equivalently redefined as $\underline{\mathbf{B}}^{\mathrm{R}}\left(q^{-1}\right)=$ $\underline{\boldsymbol{\beta}}^{\mathrm{T}}\left(q^{-1}\right)\left[\underline{\mathbf{B}}\left(q^{-1}\right) \underline{\boldsymbol{\beta}}^{\mathrm{T}}\left(q^{-1}\right)\right]^{-1}$.

The polynomial form of $\sigma$-inverse can be downgraded to parameter one for $\underline{\mathbf{B}}\left(q^{-1}\right)=\underline{\mathbf{b}}_{\mathbf{0}}$, and have the parameter $\sigma$-inverse formula simplified do the following form

$$
\underline{\mathbf{B}}^{\mathrm{R}}\left(q^{-1}\right)=\boldsymbol{\beta}^{\mathrm{T}}\left[\underline{\mathbf{B}}\left(q^{-1}\right) \boldsymbol{\beta}^{\mathrm{T}}\right]^{-1},
$$

with parameter degrees of freedom $\boldsymbol{\beta}$. Some issues concerning $\boldsymbol{\beta}$ having polynomial matrices can be found in Ref. [5].

Remark 3. For $\underline{\boldsymbol{\beta}}\left(q^{-1}\right)=\underline{\mathbf{B}}\left(q^{-1}\right)$ the $\sigma$-inverse specializes to $T$-inverse.

Remark 4. In Eqn. (8) it should rather be written $\mathbf{B}^{\mathrm{R}}$ instead of $\underline{\mathbf{B}}^{\mathrm{R}}\left(q^{-1}\right)$, as well as $\mathbf{B}$ instead of $\underline{\mathbf{B}}\left(q^{-1}\right)$.

Having the notion of polynomial inverses and their parameter representations, we are able to implement the $T$-, $\sigma$-inverses into the fractional-order perfect control law as in Eqn. (6), which is needed to run the simulation study. In the simulation study $T$-inverse is used, $\sigma$ inverse is applied only for cases in which $T$-inverse generates unstable control inputs. An $H$-inverse, basing on SVD decomposition, with parameter degrees of freedom $\mathbf{L}$ [3]

$$
\mathbf{A}^{\mathrm{R}}=\left(\mathbf{V}^{\mathrm{T}}\right)^{-1} \boldsymbol{\Sigma}^{\mathrm{R}} \mathbf{U}^{-1},
$$

where $\boldsymbol{\Sigma}^{\mathrm{R}}=\left[\begin{array}{c}\boldsymbol{\Sigma}_{\mathrm{m}}^{-1} \\ \mathbf{L}\end{array}\right]$, can be used instead. However, application of $\sigma$-inverse with parameter degrees of freedom $\boldsymbol{\beta}$ is more convenient solution, specifically.

\section{Energy-based improvement in fractional-order perfect control}

The aim of the study is to present that even minor change in the order of the system may lead to the increase of perfect control robustness. The manipulation of parameter $\alpha$, where $\alpha=1$ for classical systems i.e. integer-order systems, can directly affect the perfect control counteracting the undesirable rumbling. To 
accomplish the objectives of the study, the measures enabling evaluation how the alteration of the parameter $\alpha$ influence the robustness of the perfect control in terms of energy, have to be establish. Moreover the value for which the perfect control energy and state vector is close to the minimum, has to be determined. The introduced below indices are basing on the perfect control and state vector energy. To measure the impact of perfect control energy $E(\mathbf{u}(k))$ and the state vector energy $E(\mathbf{x}(k))$ the following performance indices are introduced, respectively

$$
\begin{aligned}
& E(\mathbf{u}(k))=\sum_{k=0}^{\infty}\left[\mathbf{u}(k)-\mathbf{u}_{\text {stab }}(k)\right]^{\mathrm{T}}\left[\mathbf{u}(k)-\mathbf{u}_{\text {stab }}(k)\right], \\
& E(\mathbf{x}(k))=\sum_{k=0}^{\infty}\left[\mathbf{x}(k)-\mathbf{x}_{\mathbf{s t a b}}(k)\right]^{\mathrm{T}}\left[\mathbf{x}(k)-\mathbf{x}_{\text {stab }}(k)\right] .
\end{aligned}
$$

The $\mathbf{u}_{\text {stab }}$ and $\mathbf{x}_{\text {stab }}$ are the stable prefect control input and state vectors, accordingly.

\section{Simulation study}

Let us take into account the integer order system $\mathrm{S}(\mathbf{A}, \mathbf{B}, \mathbf{C})$ in state-space, where $\mathbf{A}=\left[\begin{array}{cc}0.25 & -0.75 \\ -0.05 & -0.80\end{array}\right]$, $\mathbf{B}=\left[\begin{array}{ll}-0.75 & 0.90 \\ -0.10 & 0.30\end{array}\right], \quad \mathbf{C}=\left[\begin{array}{ll}0.90 & -0.90\end{array}\right]$ and initial state vector $\mathbf{x}_{\mathbf{0}}=\left[\begin{array}{ll}-4.00 & 9.00\end{array}\right]$. The system is chosen because of rumblings in control, which make it difficult to stabilize. Simulation study is carried out in Matlab/Simulink environment with the time horizon $k_{\max }=200$ embracing the subsequent simulations for several values of fractional order difference parameter $\alpha$ modified with step 0.05 . For every simulation the output remains at the reference/setpoint for $k \geq d=1$, under the stabilizing fractional-order perfect controls (and $\mathbf{x}(k)$ runs). The $E(\mathbf{u}(k))$ and $E(\mathbf{x}(k))$ indices are computed for each simulation and garnered in the Tables 1 and 2 .

Tables 1 and 2 present results of simulation for values of parameter $\alpha \geq 1$, for $\alpha<1$ the perfect control become unstable. The results of simulations show that slight increase in the order of perfect control (between $1<\alpha \leq 1.15$ ) affect the improvement in perfect control robustness in terms of control energy needed for its stabilization. Some results of simulation study are presented in Figs. 1-6. It can be observed that significant increase in perfect control robustness is obtained for $\alpha \approx 1.15$, where the value $E(\mathbf{u}(k))$ is close to minimum. There is also decline in $E(\mathbf{x}(k))$ value, although for mentioned $\alpha$ does not reach a minimum value. Moreover the Fig. 5 may suggest that for $\alpha=1.20$ there is still improvement in perfect control robustness. In fact the value $E(\mathbf{u}(k))$, interpreted as the energy of perfect control, increases, which means that system go beyond its optimum point.

It should also be strongly indicated that the robust fractional-order perfect control strictly dedicated to noninteger order plants described by the Grünwald-Letnikov fractional difference operator can be obtained in a different way. Another example could be an application of various nonunique right inverses of $\mathrm{CB}$ product (for example see Eq. (6)). Due to special selected degrees of freedom we can significantly improve the robustness of this control strategy. The issue corresponds with the perfect control design of multivariable integer-order systems.

Tab. 1 - summary of the simulation study results for $E(\mathbf{u}(k))$.

\begin{tabular}{|c|r|r|r|c|c|}
\hline$\alpha$ & \multicolumn{1}{|c|}{$\mathbf{u}_{\mathbf{1}}$} & \multicolumn{1}{c|}{$\mathbf{u}_{\mathbf{2}}$} & $E(\mathbf{u}(k))$ & $\mathbf{u}_{\mathbf{1 s t a b}}$ & $\mathbf{u}_{2 \text { stab }}$ \\
\hline 1.00 & 46.1374 & 39.3123 & 85.4497 & -0.6182 & 0.5707 \\
\hline 1.05 & 14.2739 & 12.1624 & 26.4363 & -0.6180 & 0.5705 \\
\hline 1.10 & 8.6567 & 7.3761 & 16.0328 & -0.6179 & 0.5704 \\
\hline 1.15 & 8.2402 & 7.0213 & 15.2615 & -0.6179 & 0.5704 \\
\hline 1.20 & 10.4085 & 8.8688 & 19.2772 & -0.6179 & 0.5704 \\
\hline 1.25 & 14.4960 & 12.3516 & 26.8476 & -0.6179 & 0.5704 \\
\hline
\end{tabular}

Tab. 2 - summary of the simulation study results for $E(\mathbf{x}(k))$.

\begin{tabular}{|c|r|r|r|c|c|}
\hline$\alpha$ & \multicolumn{1}{|c|}{$\mathbf{x}_{\mathbf{1}}$} & $\mathbf{x}_{\mathbf{1}}$ & $E(\mathbf{x}(k))$ & $\mathbf{x}_{1 \text { stab }}$ & $\mathbf{x}_{\text {2stab }}$ \\
\hline 1.00 & 562.7609 & 614.9315 & 1177.6924 & 1.2070 & 0.0959 \\
\hline 1.05 & 179.6233 & 231.7935 & 411.4168 & 1.2070 & 0.0959 \\
\hline 1.10 & 96.0203 & 148.1910 & 244.2113 & 1.2070 & 0.0959 \\
\hline 1.15 & 62.0046 & 114.1754 & 176.1800 & 1.2070 & 0.0959 \\
\hline 1.20 & 45.5737 & 97.7445 & 143.3183 & 1.2070 & 0.0959 \\
\hline 1.25 & 37.7172 & 89.8878 & 127.6050 & 1.2070 & 0.0959 \\
\hline
\end{tabular}

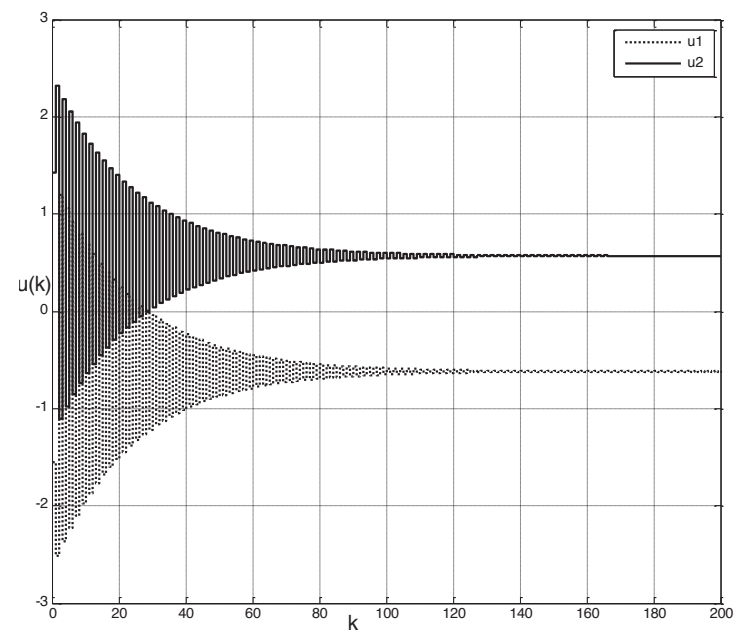

Fig. $1-\mathbf{u}(k)$ for $\alpha=1, \mathrm{k}=200$.

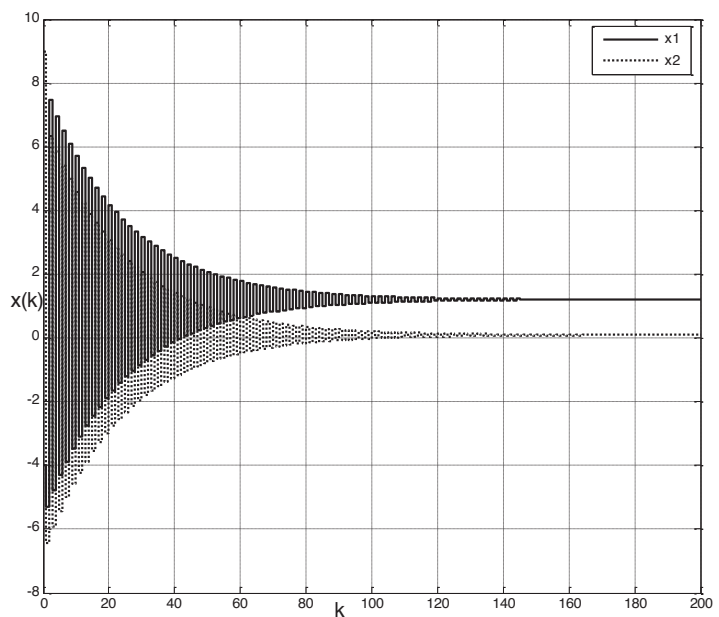

Fig. $2-\mathbf{x}(k)$ for $\alpha=1, \mathrm{k}=200$. 


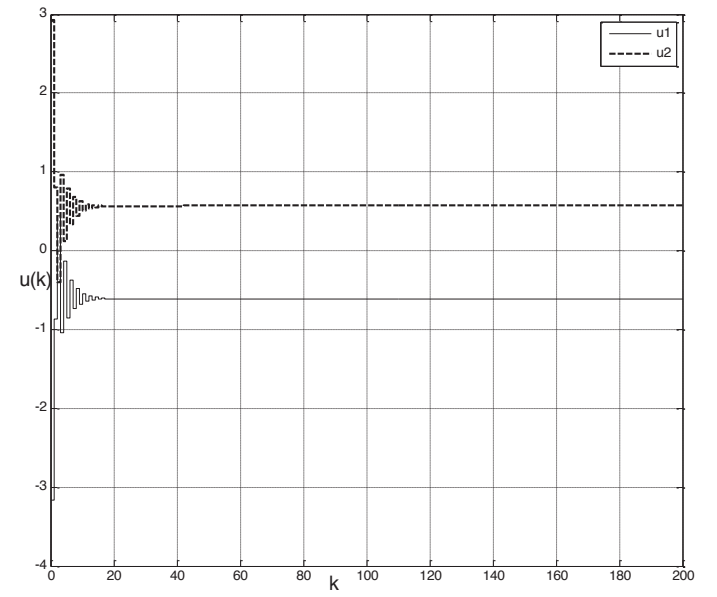

Fig. $3-\mathbf{u}(k)$ for $\alpha=1.15, \mathrm{k}=200$.

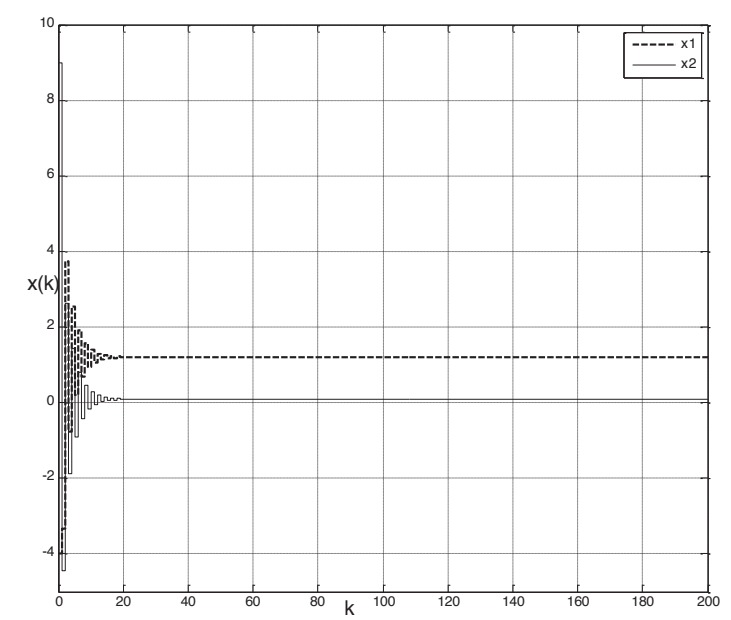

Fig. $4-\mathbf{x}(k)$ for $\alpha=1.15, \mathrm{k}=200$.

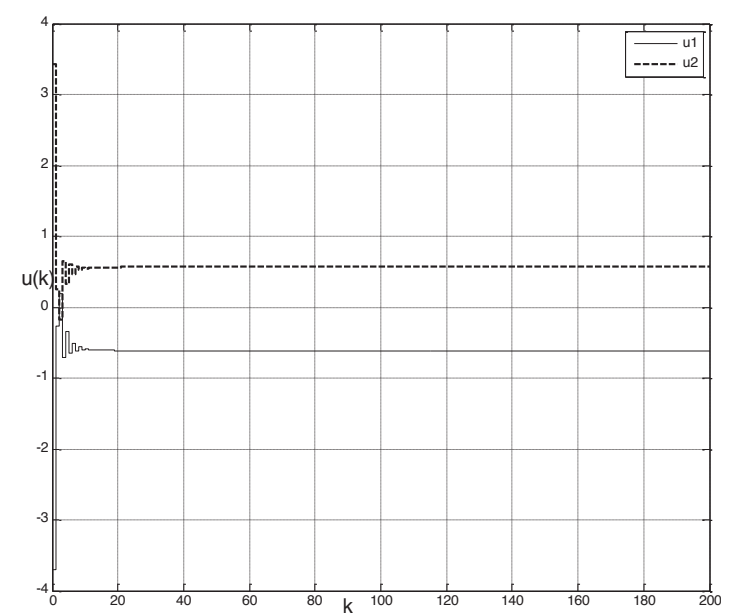

Fig. $5-\mathbf{u}(k)$ for $\alpha=1.20, \mathrm{k}=200$.

Note that for fractional-order systems of Eqns. (1) the output remains at the reference/setpoint for $k \geq d=1$ even under unstable perfect control, this is a main feature of this control strategy. Nevertheless, due to an application of different tools we can immediately obtain the minimum phase behavior of non-integer order plants. It can only be done for nonsquare systems having different number of input and output variables in contrast to the square ones where the mechanism of nonuniqueness does not exist.

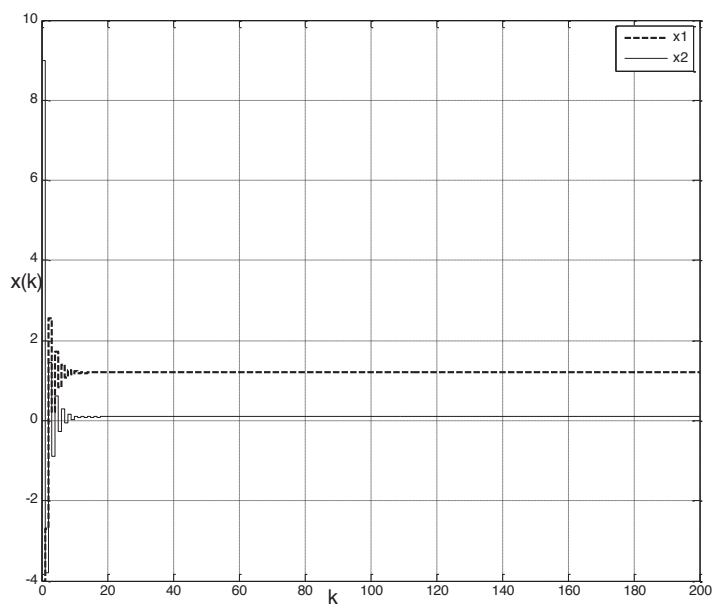

Fig. $6-\mathbf{x}(k)$ for $\alpha=1.20, \mathrm{k}=200$.

\section{Conclusions}

In the paper the new approach to minimize the energy of perfect control and growth of the system robustness by application of fractional-order model has been presented. The simulation examples of multivariable discrete-time system in state-space framework have been carried out in Matlab/Simulink environment. The results of simulation study, evaluated using the introduced authors' indices, show the decrease of energy required to stabilize the system inputs as well as decline in state vector energy. That confirms the correctness of the presented strategy and demonstrates the potential of proposed method in reducing control energy. The future study can be focused on search of the analytical formula that proofs the results obtained in the simulation tasks.

\section{References}

1. W.P. Hunek, Towards a General Theory of Control Zeros for LTI MIMO Systems, Opole Univ. of Tech. Pre. (2011)

2. W.P. Hunek, K.J. Latawiec, P. Majewski, P. Dzierwa, An application of a new matrix inverse in stabilizing state-space perfect control of nonsquare LTI MIMO systems, Proc. of the 19th IEEE Int. Conf. on Met. and Mod. in Auto. and Rob. (MMAR), 451-455 (2014)

3. W.P. Hunek, New SVD-based matrix H-inverse vs. minimum-energy perfect control design for statespace LTI MIMO systems. Proc. of the 20th IEEE Int. Conf. on Sys. Theory, Cont. and Comp. (ICSTCC'16), Sinaia, Romania, pp. 14-19, (2016)

4. W.P. Hunek, An application of polynomial matrix $\sigma$ inverse in minimum-energy state-space perfect control of nonsquare LTI MIMO systems, Proc. of the 20th Int. Conf. on Met. and Mod. in Auto. and Rob. (MMAR), 2521-255 (2015)

5. W.P. Hunek, An application of new polynomial matrix $\sigma$-inverse in minimum-energy design of robust minimum variance control for nonsquare LTI 
MIMO systems. Proc. of the 8th IFAC Symp. on Robust Cont. Design (ROCOND'2015), Bratislava, Slovakia, pp. 149-153, (2015)

6. K. J. Latawiec, The Power of Inverse Systems in Linear and Nonlinear Modelling and Control. (Opole Univ. of Tech. Press, Opole, 2004)

7. W. P. Hunek, Ł. Wach, Towards a New Stability Criterion for Fractional-Order Perfect Control of LTI MIMO Discrete-Time Systems in State-Space Proc.of 3rd IEEE Int. Conf. on Cyb. CYBCONF2017, Exeter, UK, pp. 134-139, (2017)

8. Ł. Wach Ł., W.P. Hunek, JAMRIS 10, (2016)

9. A. Ben-Israel, T.N.E. Greville, Generalized Inverses, Theory and Applications (Springer-Verlag, 2 ed., New York, 2003)

10. Ł. Wach, W.P. Hunek, Perfect control for fractional-order multivariable discrete-time systems, Theoretical Developments and Applications of NonInteger Order Systems, Lecture Notes in Electrical Engineering 357, Proc. of the 7th Conf. on Non-int. Ord. Calc. and its Appl. (RRNR'2015), Szczecin, Poland, pp. 233-237, (2015)

11. D. Sierociuk, Estimation and control of discrete fractional order state space systems Ph.D. dissertation, Warsaw University of Technology, Warsaw, Poland, 2007 (in Polish) 